\title{
Forecasting Wind Speed using Artificial Neural Networks - A Case Study of a Potential Location of Saudi Arabia
}

\author{
Yunus Parvej Faniband, ${ }^{1,}$ and S. M. Shaahid ${ }^{2}$ \\ ${ }^{1}$ Center for Communication \& IT Research, Research Institute, King Fahd University of Petroleum \& Minerals, Saudi Arabia \\ ${ }^{2}$ Center for Engineering Research, Research Institute, King Fahd University of Petroleum \& Minerals, Dhahran, Saudi Arabia
}

\begin{abstract}
The growing concerns regarding the depletion of oil/gas reserves and global warming have made it inevitable to seek energy from wind and other renewable energy resources. Forecasting wind speed is a challenging topic and has important applications in the design and operation of wind power systems, particularly grid connected renewable energy systems, and where forecasting wind speed helps in manipulating the load on the grid. Modern machine learning techniques including neural networks have been widely used for this purpose. As per literature, various models for estimating the hourly wind speed one hour ahead and the hourly wind speed data profile one day ahead have been developed. This paper proposes the use of Artificial Intelligence methods (AI) which are most suitable for the prediction and have provided best results in many situations. AI method involves nonlinear (or linear) and highly complex statistical relationships between input and output data, such as neural networks, fuzzy logic methods, Knearest Neighbors algorithm (KNN) and Support Vector Machine (SVM). AI methods are promising alternatives for predicting wind speed and understanding the wind behavior for a particular region. In the present study (as a case-study), hourly average wind speed data of 13 years (1970-1982) of Qaisumah, Saudi Arabia has been used to evaluate the performance of ANN model. This data has been used for training the neural network. ANN is trained multiple times with different number of hidden neurons to forecast accurate wind speed. The efficiency of proposed model is validated by predicting wind speed of the Qaisumah region with the measured data. Mean Square Error (MSE) and mean absolute percentage error (MAPE values) for proposed model are found to be 0.0912 and $6.65 \%$ respectively.
\end{abstract}

\section{Introduction}

Artificial Intelligence (AI) is a technology that aims to simulate or extend human intelligence abilities by developing theories, methods, techniques, and applications. Machine Learning(ML) make use of large data sets in assisting machines to learn, rather than using conventional hard coded instructions. Higher computing power of the modern computers enable ML to quickly process large data sets. Artificial Neural Networks (ANNs) also known as Deep Learning (DL) is a subset of ML that assist to learn superior representations automatically by adopting supervised or unsupervised strategies [1] [2].

ANN is a complex mathematics model influenced by the model of human brain neurons, which are characterized to understand and learn by observing the human brain. Supervised Learning [3] [4] [5] is characterized with labelled data sets that have inputs and anticipated outputs. Unsupervised learning [6] strategy operates with data sets that has no specified structure.

One of the principal benefits of ANN is their capacity to generalize. This means that, once the ANN is trained through the data presented to them (a sample), it can correctly infer data from the unseen proportion of a population of a dataset. Generalization of the ANN is ability to handle unseen data even if the sample data contain noisy information.

Forecasting process is a perfect environment for neural networks, as it is carried out through prediction of future behavior (the unperceived proportion) from patterns of past behavior [7]. ANNs, as opposed to conventional model-based approaches, are self-adaptive reliable predictive models [8]. ANN exhibit multiple exclusive characteristics that render them ideal for a forecasting mission. Often if the underlying relationships remain obscure or challenging to explain, ANNs benefit from learning and catch implicit functional relationship among the data. ANNs are thus ideally to assist for problems, where solutions need information that is challenging to determine, but has adequate data or insights.

A prediction or forecasting model is based on the presumption that an underlying (known or unknown) relationship prevail between the inputs and the outputs (the future values). Due to the complexity of the real

\footnotetext{
* Corresponding author: yparvej@kfupm.edu.sa
} 
prediction system, where inputs have the past values of the time series and/or other relevant variables, tradition statistical forecasting models have limitations estimating the underlying function. ANNs can be an ideal solution to define this function.

Furthermore ANNs are nonlinear statistical data modeling tools, whereas Forecasting has long been the domain of linear statistics. Traditional approaches to time series forecasting models are based on the assumption that the time series under analysis are generated from linear processes. The benefits of linear models are that they are simple to describe and ability to fully understand and analyze to any great extent. However the limitation of the linear models lies in that most real world systems that are often nonlinear. Compared to the model-based nonlinear methods, the nonlinear data-driven approaches of ANN are efficient to implement nonlinear modeling without previous knowledge about the complex relationships between input and output variables. ANNs are thus ideally flexible modeling tools for forecasting.

Presently, ANNs are employed in a broad variety of tasks, ranging in many different fields of science, industry and business. Several articles are available in literature on ANNS.

There are several studies to conclude that ANNs provide ideal alternative approach compared to traditional methods. Guoqiang Zhang et al not only presented a detail review of ANN applications in forecasting with focus on insights on ANN modeling issues, synthesis of published research in this area, future research directions, but also reported relative performance of ANNs over traditional statistical methods [1].

In order to investigate the effectiveness of using ANNs in the area of energy load forecasting at individual building level, Amarasinghe et al. [2] discuss the approach using historical loads by using a benchmark data set of electricity consumption for a single residential customer. Compared with approach of Long Short-Term Memory (LSTM), the ANN based methods proposed by Authors, considerably outperformed.

Precise load forecasting is needed for utilities to predict their customer's demands (electric load). Since last decade, considerable attention has been focused on use of ANNs to model load, since ANNs are ideal for modeling an unspecified non-linear relation between load and meteorological variables. In the domain of Weather forecasts ANNs are extensively used. In a study conducted by Taylor and Buizza [3], to load forecasting for lead times from 1 to 10 days ahead, using the weather ensemble predictions predictions in the application of ANN. Compared with the forecast that produced using traditional methods of weather forecasts, the results using the ANN approach resulted in more accurate average of the load forecast.

Recent studies also focused on the domain of coastal and marine applications, where forecasting of wave characteristics is critical. Apart from employing
ANN as one of the most common soft computing methods, this domain uses various approaches like empirical, statistical and soft computation and data learning methods. Several studies are performed using ANN to estimate wave height and average wave time values from the corresponding wind speed values and related characteristics [4] [5]. The values of significant wave height and mean wave period have been estimated by Deo and others [5] using ANN. Their ANN model used corresponding values of wind speed and related properties, and trained using three data sets and employing different algorithms. The results from this Author show that an adequately trained network could produce satisfactory results in deep water, open wide areas and and even when the period of sampling and prediction is long, for example a week. In their research, Kamranzad et al. [4] used ANN to predict wave height in the Persian Gulf for the next 3, 6, 12 and 24 hours. Some studies focus on Short-term wind power forecasting using different approaches like empirical mode decomposition and radial basis function neural networks (RBFNN) [7], while the studies [8] [9] used LSTM based recurrent neural network (RNN) model. The paper [10] provides a case study of the effects of wind turbine wake losses on the assessment of wind resources in cold region. Sameer et al. [11] are focusing on quantification of the uncertainty affecting the forecasts of wind energy generation made available by a series of artificial networks.

In the recent past, there has been great deal of attention in applications of ANNs in the areas of financial statistics and exchange rates. Emi Nakamura [6] has evaluated the usefulness of ANNs for inflation forecasting. ANNs have been found to outperform autoregressive models. Petnahazi [12] in his study explores and describes several aspects of the application of ANNs to time series forecasting. ANNs have been found to be much more flexible and much better suited to time series forecasting than the linear models usually applied.

Weather data is not only nonlinear but also follow extremely irregular trend and ANNs has evolved into a better technique to analyze systemic connections between the different entities. Artificial neural models are ideal for weather forecasting in complex atmospherical environments with a supervised learning framework. In his work, Subhajini [13] has investigated the development of an effective ANN model for weather prediction (for one day in advance), which is efficient, reliable and accurate. The study used a training dataset with a total of ten years of data collected. Kumar et al.[14] evaluated ANN's applicability by designing effective and reliable nonlinear weather prediction forecasting models. The model furcates maximum temperature for 365 days a year. In another study Suksri et al. [15] suggested the use of Fireworks Algorithm to train the ANNs and predict a daily average temperature based on different parameters. The results show that the strategy proposed was beneficial. In order to predict maximum and minimum temperature, Kumar et al.[16] used ANNs. The results demonstrated the potential for successful application of 
the ANNs model based on the Multilayer Perceptron (MLP).

In the light of the above literature review, currently no studies are available on wind speed forecasting of Qaisumah, Saudi Arabia using artificial neural networks. This is the motivation of the study. In the present study (as a case-study), hourly average wind speed data of Qaisumah, Saudi Arabia of about hourly average of 13 years (1970-1982) has been used to evaluate the performance of ANN model.

\section{Background Information of study area and data}

The Kingdom of Saudi Arabia (KSA) is basically a desert land with hot summers and cold winters. Climatic conditions determine availability of wind energy at a given site. Wind power systems are characterized by availability of wind speed resources. Qaisumah is one of the potential wind resource location. The long term wind speed data used in the present study covers the period 1970-1982 [17] and is shown in Fig 1. This data has been used for training the neural network.

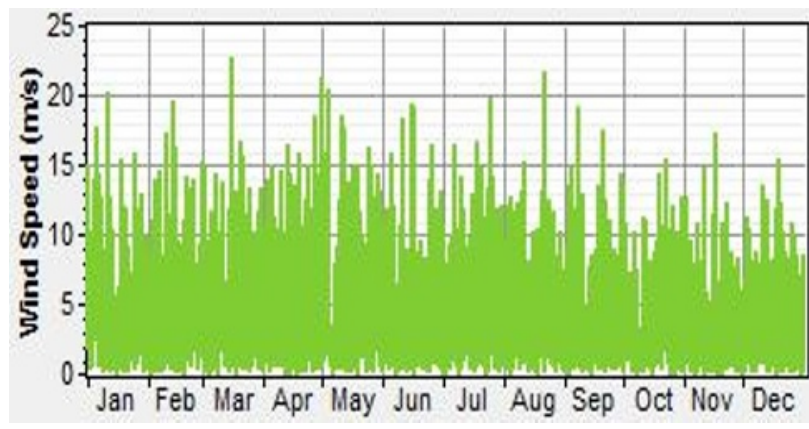

Fig. 1. Long-term (1970-1982) average wind speed for different months of Qaisumah, Saudi Arabia

\section{Methodology}

In this section dataset used for ANN model and architecture of ANN model is described briefly. Total 8760 (hourly average of 13 years) data samples of Qaisumah, Saudi Arabia are used for training the ANN.

\subsection{Architecture of ANN model:}

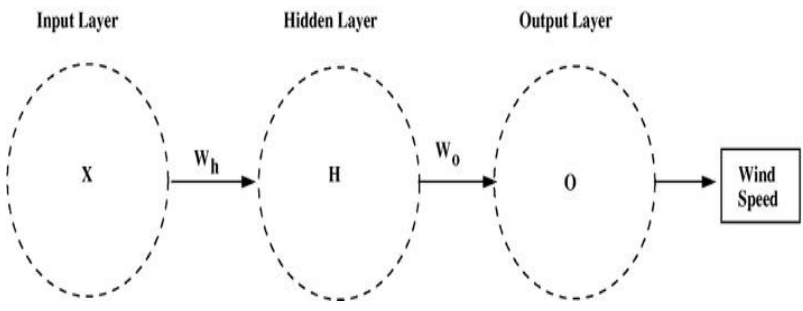

Fig. 2. Architecture of ANN model
ANN is a computational tool consisting of simple parallel elements commonly known as neurons, which mimic brain and nervous system functioning of humans. They are composed of three layers (input, hidden and output layers) inter-connected with each other. It is used as a replacement for logistic regression, a statistical technique that predicts a variable result based upon predetermined input parameters.

Artificial neural networking of this paper is implemented using MathWorks Neural Net Fitting tool. Input Layer holds the data ANN model that will be trained on. Each neuron in the input layer represents a unique attribute in the dataset such as wind speed, earth temperature, relative humidity and elevation. In order to map the input data and target value for a two-layer feed forward neural network, the Levenberg Marquardt Back Propagation algorithm is used. Hidden Layer is positioned between the input and output layers and uses the active function before the results are transferred. Of the total of 8,760 available samples, 7008 samples are used for training and the remaining 1752 for testing purposes. These samples are divided into testing and validations. $20 \%$ of the 1752 samples used for analysis and the same was used for validation in this data set. Hidden layers typically are completely connected layers for traditional networks, with each neuron receiving input of all the neurons of the previous layer and transmitting its output to all the neurons of the next level. Training of Neural network is achieved by mapping between numeric inputs and numeric targets, through different number of hidden neurons. The main input variable is the measured wind speed at this location. The other parameters than may be considered for the input include earth temperature, relative humidity, elevation etc. Wind speed is the target.

The two parameters that are used to calculate the Accuracy of the wind speed forecasting are: Mean Square Error (MSE) and Mean Absolute Percentage Error (MAPE), which are evaluated using following equations.

$$
M S E=\frac{1}{T} \sum_{i=1}^{T}\left[\left(W S i_{\text {measured }}-W S i_{\text {predicted }}\right)\right]^{2}
$$

$$
M A P E=\frac{1}{T} \sum_{i=1}^{T}\left|\frac{\left(W S i_{\text {measured }}-W S i_{\text {predicted }}\right)}{W S i_{\text {measured }}}\right| * 100
$$

\section{Results and discussion}

Fig 3 shows the comparison between wind speed measured and wind speed forecasted. The ANN was trained using 8760 samples, however for the sake of brevity, Fig 3 shown only 300 records. The wind speed values predicted by the ANN model are very similar to the measured wind speed values. For the proposed ANN model, the average square error (MSE) and mean absolute percentage error (MAPE) were found to be 
0.0912 and $6.65 \%$ respectively. As per the literature (Lewis [18]), when MAPE is below 10\% then model is predictively accurate, and when the MAPE ranges from $10 \%$ to $20 \%$, model predictability is good. The proposed ANN method therefore has a high accuracy of prediction.

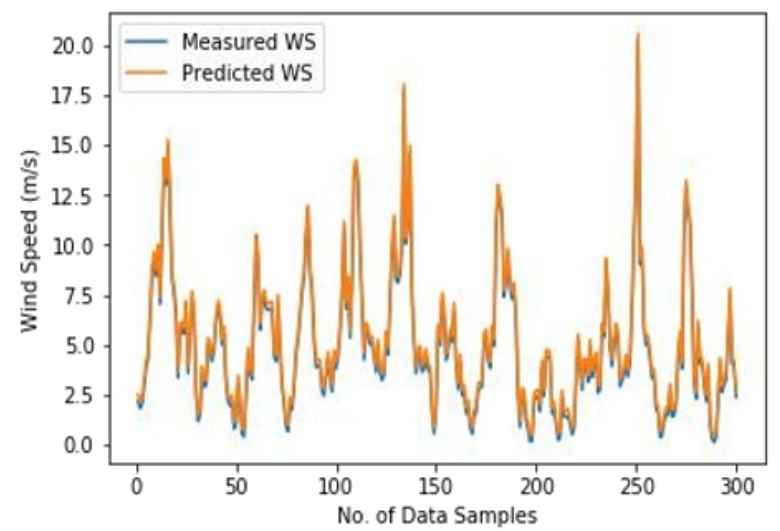

Fig. 3. Graph showing comparison of measured wind speed and predicted wind speed.

\section{Conclusions}

The current study proposes an artificial neural network for long-term wind speed predictions. Previously observed measured wind speed at this location is used as input variable. The long term hourly average wind speed data used in the present study covers the period 19701982 of Qaisumah, Saudi Arabia. The neural network is trained with a different number of invisible neurons and the best performance is found when the artificial neural network with 29 hidden neurons is trained. Mean square error (MSE) and mean absolute percent error (MAPE) values of 0.0912 and $6.65 \%$ respectively are obtained for the proposed model. The ANN model's efficiency is validated by comparing the measured wind speed data with the predicted wind speed data. Future work on this topic shall include comparison of present results with other models available in literature.

\section{Acknowledgement}

The authors acknowledge the support of Center for Communication \& IT Research and Center for Engineering Research, Research Institute, King Fahd University of Petroleum and Minerals for this research work.

\section{References}

1. G. Zhang, B. E. Patuwo, and M. Y. Hu, "Forecasting with artificial neural networks:: The state of the art," Int. J. Forecast., vol. 14, no. 1, pp. 35-62, (1998).

2. K. Amarasinghe, D. L. Marino, and M. Manic, "Deep neural networks for energy load forecasting," IEEE Int. Symp. Ind. Electron., pp. 1483-1488, (2017)

3. J. W. Taylor and P. E. McSharry, "Univariate methods for short-term load forecasting," Advances in Electric Power and Energy Systems: Load and
Price Forecasting. Wiley Online Library, pp. 17-39, (2017).

4. B. Kamranzad, A. Etemad-Shahidi, and M. H. Kazeminezhad, "Wave height forecasting in Dayyer, the Persian Gulf," Ocean Eng., vol. 38, no. 1, pp. 248-255, (2011).

5. M. C. Deo, A. Jha, A. S. Chaphekar, and K. Ravikant, "Neural networks for wave forecasting," Ocean Eng., vol. 28, no. 7, pp. 889-898, (2001).

6. E. Nakamura, "Inflation forecasting using a neural network," Econ. Lett., vol. 86, no. 3, pp. 373-378, (2005).

7. Z.-W. Zheng, Y.-Y. Chen, X.-W. Zhou, M.-M. Huo, B. Zhao, and M. Guo, "Short-term wind power forecasting using empirical mode decomposition and RBFNN," Int. J. Smart Grid Clean Energy, vol. 2, no. 2, pp. 192-199, (2013).

8. L. Shijian, H. Yongjun, and L. Fuchao, "Application of the combined model in short-term wind power forecasting.", International Journal of Smart Grid and Clean Energy, vol. 5, no. 3, July 2016

9. U. Cali and V. Sharma, "Short-term wind power forecasting using long-short term memory based recurrent neural network model and variable selection," Int. J. Smart Grid Clean Energy, vol. 8, no. 2, pp. 103-110, (2019).

10. J. Y. Jin, R. Ghani, and M. S. Virk, "Wind turbine wake effects on wind resource assessments-A case study." International Journal of Smart Grid and Clean Energy, vol. 9 , no. 1, January (2020).

11. S. Al-Dahidi, P. Baraldi, E. Zio, and M. Lorenzo, "Quantification of uncertainty of wind energy predictions," in Proc. 3rd Int. Conf. Syst. Rel. Saf., 2018, pp. 1-5, (2018).

12. G. Petneházi, "Recurrent neural networks for time series forecasting," arXiv Prepr. arXiv1901.00069, (2019).

13. A. Subhajini and others, "Application of Neural Networks in Weather Forecasting," (2014).

14. K. Abhishek, M. P. Singh, S. Ghosh, and A. Anand, "Weather Forecasting Model using Artificial Neural Network," Procedia Technol., vol. 4, pp. 311-318, (2012).

15. S. Suksri and W. Kimpan, "Neural network training model for weather forecasting using fireworks algorithm," in 2016 International Computer Science and Engineering Conference (ICSEC), pp. 1-7, (2016).

16. N. Kumar and G. K. Jha, "Time Series ANN Approach for Weather Forecasting," Int. J. Control Theory Comput. Model., vol. 3, no. 1, pp. 19-25, (2013).

17. J. M. Al-Ansari, H. Bakhsh, and K. I. Madni, "Wind energy atlas for the kingdom of Saudi Arabia," King Fahd Univ. Pet. Miner. Press. Dhahran, (1986).

18. C. Lewis, "International and Business Forecasting Methods Butterworths: London," (1982). 